\title{
Separación de funciones en el Sistema de Protección Social en Salud, México 2009:
} avances y retos

\author{
Luz María González-Robledo, PhD, (1) Gustavo Nigenda, PhD, (2)
}

María Cecilia González-Robledo MC, ${ }^{(2)}$ Michael Reich PhD.(3)

\begin{abstract}
González-Robledo LM, Nigenda G, González-Robledo MC, Reich M Separación de funciones en el Sistema de Protección Social en Salud, México 2009: avances y retos. Salud Publica Mex 20 I ;;53 supl 4:S416-S424.
\end{abstract}

\section{Resumen}

Objetivo. Analizar los avances y retos de la separación de funciones en el Sistema de Protección Social en Salud en México. Material y métodos. Investigación evaluativa realizada en 2009. Se incorporaron nueve entidades federativas y la Comisión Nacional de Protección Social en Salud. Se realizaron entrevistas semiestructuradas a informantes clave y análisis documental. Resultados. El principal avance es la creación de los Regímenes Estatales de Protección Social en Salud (REPSS), con una función de intermediación entre los usuarios y los prestadores de servicios de salud. Éstos se constituyen como instancias del nivel estatal encargadas de administrar los recursos financieros y conformar y coordinar la red de prestación de servicios de atención médica. No obstante, la mayoría de los REPSS estudiados se encuentran en un estado de inercia que les impide cumplir adecuadamente con las funciones marcadas por la ley. Conclusión. Persisten obstáculos normativos, técnicos, políticos y gerenciales para cumplir con la separación de funciones.

Palabras clave: financiación de la salud; gestión en salud; México
González-Robledo LM, Nigenda G,

González-Robledo MC, Reich M

Separation of functions in the System of Social Protection

in Health, Mexico 2009: progress and challenges.

Salud Publica Mex 20II;53 suppl 4:S4I6-S424.

\section{Abstract}

Objective. To evaluate advancements and challenges in the separation of functions within Mexico's System of Social Protection in Health. Material and Methods. A 2009 evaluation study involving nine states and the National Commission for Social Protection in Health was carried out via semi-structured interviews with key actors and literature analysis. Results. The main advancement has been the creation of the State Regimens for Social Protection in Health (REPSS in Spanish) which act as intermediaries between users and health service providers, making these state-level entities responsible for both managing financial resources and shaping and coordinating the health care delivery network. However, most of the REPSS studied were found to be in a state of inertia, leading to inadequate compliance with legally mandated functions. Conclusion. Normative, technical, political and managerial obstacles persist, impeding the successful separation of functions.

Key words: health financing; health management; Mexico

(I) Universidad Autónoma del Estado de Morelos. Cuernavaca, Morelos, México.

(2) Instituto Nacional de Salud Pública. Cuernavaca, Morelos, México.

(3) Universidad de Harvard. Cambridge, MA, EUA.

Fecha de recibido: 17 de agosto de 2010 - Fecha de aceptado: 18 de mayo de 201। Autor de correspondencia: Dra. María Cecilia González Robledo. Instituto Nacional de Salud Pública. Av. Universidad 655, Col. Santa María Ahuacatitlán. 62100 Cuernavaca, Morelos, México.

Correo electrónico: cecilia.gonzalez@insp.mx 
T a separación de las funciones de rectoría, finanLciamiento y prestación de servicios de salud ha sido una estrategia frecuentemente introducida en los procesos de reforma de los sistemas de salud, particularmente de América Latina, buscando mejorar la cobertura de salud y contribuir a la eficiencia y calidad de los servicios. ${ }^{1,2}$ En ella se establece que la responsabilidad del Estado es mantener el control de la rectoría, la regulación y el financiamiento; mientras que es posible abrir la provisión de servicios de salud a la participación plural de prestadores públicos, privados y de la seguridad social. ${ }^{3,4}$

El Sistema de Protección Social en Salud (SPSS) es una política pública inédita en la historia de la salud en México. Se estructura alrededor de un sistema de aseguramiento dirigido a la población no cubierta por las instituciones de seguridad social que tiene como fin garantizar el derecho al acceso a los servicios de salud y proteger financieramente al hogar reduciendo el gasto de bolsillo. ${ }^{5}$ La importancia del SPSS para la población radica en la certeza que provee a las familias que ante la enfermedad de uno de sus miembros no tendrán que desembolsar dinero para cubrir los servicios obtenidos y los medicamentos prescritos. Para el sistema de salud, la importancia reside en que se abre la alternativa de una reasignación de fondos federales frescos a los estados a través del pago de una prima fija por familia de acuerdo con su nivel de ingreso. ${ }^{6}$

El presente artículo documenta los avances y retos de la separación de funciones en nueve entidades federativas estudiadas, en las que se identifican aciertos, vacíos y obstáculos con el propósito de aportar evidencias para orientar el desarrollo de procesos de mejora gerenciales y operativos que optimicen el funcionamiento del sistema tanto en el ámbito estatal como en el nacional.

\section{Marco normativo que sustenta la organización} y funcionamiento del SPSS

A partir de la reforma financiera del sistema de salud en México (2003), se suscitan cambios importantes en la estructura, organización y funcionamiento del subsector público de la salud. Se incorpora a la Ley General de Salud un componente de Protección Social en Salud (PSS), cuyo propósito se orienta a garantizar el acceso efectivo, oportuno, de calidad, sin desembolso al momento de utilización y sin discriminación a los servicios de salud que satisfagan las necesidades de salud de la población. ${ }^{7}$

Se establece como responsable de la rectoría, evaluación y monitoreo a la Secretaría de Salud federal por conducto de la Comisión Nacional de Protección Social en Salud (CNPSS). ${ }^{8}$ El financiamiento se organiza a tra- vés de un componente de aseguramiento subsidiado (Seguro Popular), pasando de una lógica burocrática de subsidios a la oferta a una orientación hacia la demanda (para sustituir la asignación presupuestal histórica), mediante la transferencia de los recursos federales a los estados con base en el número de familias afiliadas. Las fuentes provienen del gobierno federal, de los gobiernos estatales y de las familias beneficiarias (incluye un principio de prepago de primas por población afiliada). ${ }^{*} \mathrm{La}$ federación aporta alrededor de 70\% del financiamiento (cuota social y aportación solidaria federal) y los estados aportan una proporción menor, cercana a 30\% de los recursos requeridos para su operación (aportación solidaria estatal). ${ }^{9-11}$

La prestación de servicios de salud es realizada primordialmente por las unidades de atención médica de los Servicios Estatales de Salud (SESA). Esta red está compuesta por centros de salud y hospitales generales quienes garantizan la prestación de los servicios esenciales de salud establecidos en el Catálogo Universal de Servicios Esenciales de Salud (CAUSES). En el caso de que los SESA (red pública) no estén en la capacidad de ofrecer alguno(s) de los servicios contemplados en el CAUSES, la ley contempla la posibilidad de comprar servicios de salud a otros prestadores de atención públicos o privados. ${ }^{12}$

En los estados, los responsables de la administración de los recursos financieros y de integrar y coordinar la red de proveedores de servicios son los Regímenes Estatales de Protección Social en Salud (REPSS), mientras que la atención es prestada por unidades públicas de salud de los SESA. Las funciones establecidas por ley para los REPSS son: a) la promoción, afiliación y tutela de los derechos de los afiliados, b) la administración y gestión de los recursos financieros, c) la compra de servicios a los prestadores, y d) la rendición de cuentas. Para que los REPSS reciban las transferencias financieras federales, el marco legal establece los requisitos que las entidades federativas deben cumplir: 1) congruencia del padrón de afiliados con los recursos transferidos; 2) comprobación del pago de la aportación solidaria estatal por parte de la entidad federativa a la CNPSS, y 3) información sobre la ejecución de los recursos. El incumplimiento de cualquiera de estas obligaciones por parte de las entidades federativas conduce a la suspensión de las transferencias económicas a los estados. ${ }^{13}$

La regulación establece explícitamente que los REPSS no prestan directamente servicios de salud. ${ }^{13} \mathrm{Su}$

\footnotetext{
* En la práctica la población afiliada no realiza una contribución significativa al financiamiento del sistema
} 
función está dirigida a la compra de servicios a través de la suscripción de acuerdos de gestión y contratos (con instituciones públicas, privadas o de la sociedad civil) y a la coordinación eficiente, oportuna y sistemática de la red de prestadores de servicios de salud (figura 1).

\section{Material y métodos}

Los resultados hacen parte de un estudio de evaluación más amplio denominado Evaluación Externa del Sistema de Protección Social en Salud, 2009 realizado por el Instituto Nacional de Salud Pública (INSP) con el apoyo financiero de la Dirección General de Evaluación del Desempeño (DGED) de la Secretaría de Salud federal. El objetivo principal se centró en la evaluación de procesos gerenciales del SPSS a través de los REPSS con el fin de obtener evidencia para apoyar y favorecer ajustes y modificaciones con vistas a mejorar la efectividad y la eficiencia del sistema.

La evaluación se basó en un diseño de investigación que incorpora técnicas de recolección y análisis de información cualitativa. Se utilizaron como estrategias de análisis la triangulación, complementariedad y expansión, buscando integrar, contrastar, confirmar y ampliar la información obtenida para construir una visión global, amplia y detallada del nivel de avance y retos de la separación de las funciones en los REPSS.

El estudio se dividió en dos etapas, la primera documental y la segunda práctica. En la etapa documental se utilizaron fuentes de información secundaria: Ley General de Salud; Reglamento de la Ley General de Salud en Materia de Protección Social en Salud; Reglamento Interno de la Comisión Nacional de Protección Social en Salud; Ley de Salud del Distrito Federal; Ley de Salud para el Estado de Campeche; Convenio Marco de Colaboración y Coordinación en Materia de Prestación de Servicios Médicos y Compensación Económica, celebrado entre las Entidades Federativas, el Distrito Federal y el Gobierno Federal; acuerdos de gestión entre el REPSS y los prestadores de salud públicos; manuales operativos de los REPSS; convenios de colaboración interinstitucional para la prestación de servicios médicos, quirúrgicos, hospitalarios, suministro de medicamentos e insumos; convenios interestatales de prestación de servicios, contratos entre el REPSS y prestadores privados y el Plan Nacional de Salud 2007-2012.

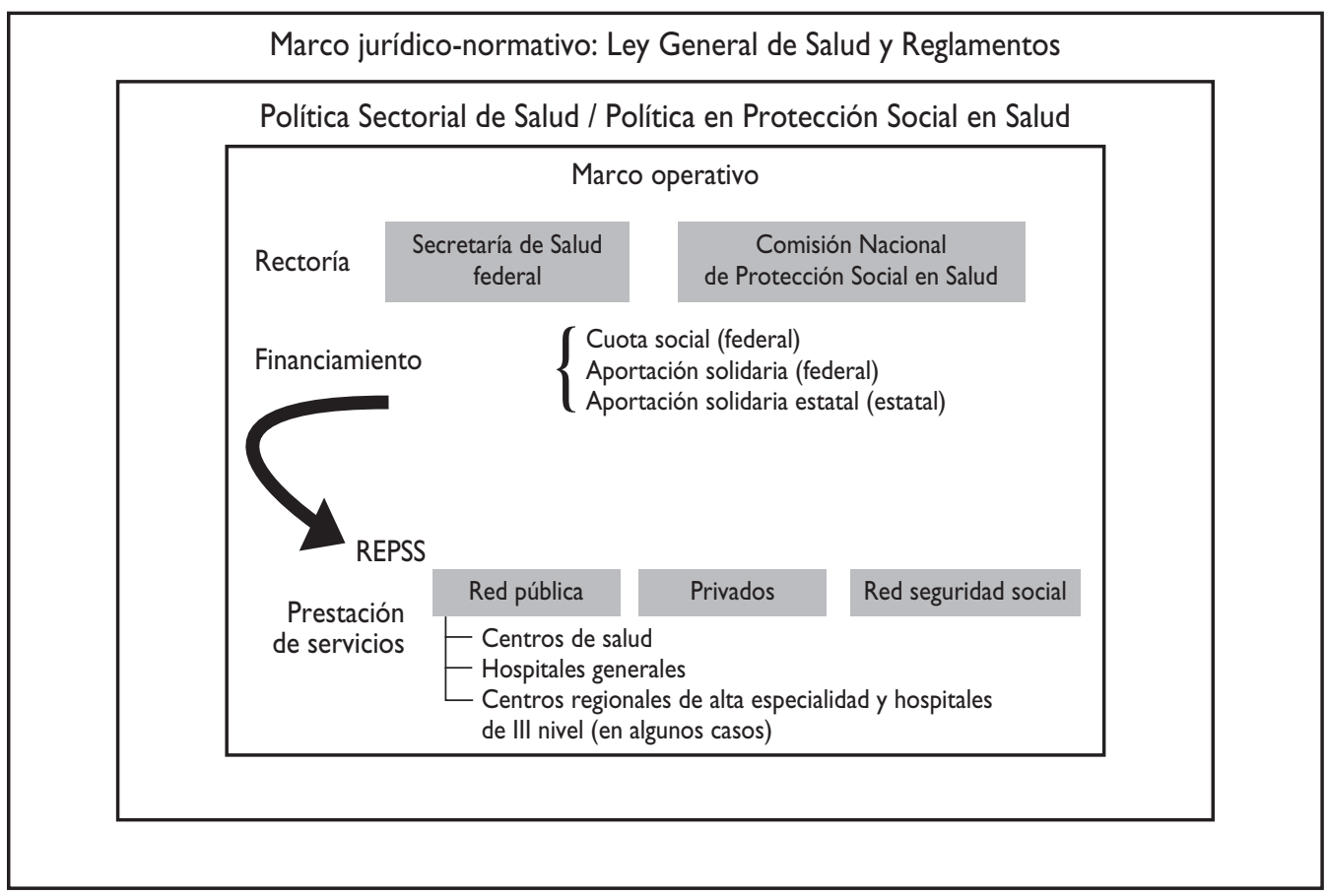

Fuente: Elaboración de los autores con información de fuentes secundarias.Estudio realizado en el Instituto Nacional de Salud Pública, México, noviembre 2009

Figura I. Estructura del Sistema de Protección Social en Salud 
En el trabajo de campo se utilizaron fuentes de información primarias (entrevistas semiestructuradas a informantes clave) y se realizó entre septiembre y noviembre de 2009. La selección de la muestra fue por criterio y el tamaño por conveniencia, con ejecución en dos etapas. En la primera se eligieron los estados a evaluar. Se seleccionaron nueve estados: Morelos, Distrito Federal, Hidalgo, Querétaro, Guerrero, Baja California, Jalisco, Campeche y Zacatecas, tomando en cuenta criterios de inclusión previamente establecidos por el equipo de investigación. En la segunda etapa se efectuó la selección de los informantes clave. El criterio principal para definirlos fue la posibilidad de encontrar respuestas -basadas en experiencia y conocimiento sustantivo-a las preguntas de las guías. Los informantes seleccionados por categoría fueron: del REPSS, el Director General, el Director de Financiamiento y Administración y el Director de Gestión de Servicios de Salud o, en su defecto, quienes ellos propusieran. En los SESA el encargado del departamento de Administración y Finanzas y, del ámbito federal, los directores de Financiamiento y Gestión de Servicios de la CNPSS. Se entrevistaron 36 funcionarios del ámbito estatal y dos del ámbito federal. Las entrevistas fueron grabadas previo consentimiento informado. La información documental se recolectó a través de los REPSS, las Secretarías Estatales de Salud, la CNPSS y medios electrónicos.

El procesamiento de la información primaria y secundaria se realizó mediante matrices sistematizadas y clasificadas según componente, tema e informante. Las categorías generales que guiaron el análisis fueron: adecuación y cumplimiento del marco jurídico que sustenta el funcionamiento del SPSS y de los REPSS, gestión y administración de los recursos financieros, conformación y coordinación de la red de prestadores de servicios de salud y principales problemas y obstáculos que debe enfrentar el REPSS para cumplir con la separación de funciones. El proyecto fue aprobado por las comisiones de Investigación, Ética y Bioseguridad del INSP.

\section{Resultados}

Para dar cumplimiento a lo establecido en el marco legal del SPSS, ${ }^{*}$ la política sectorial de salud promueve la reestructuración organizativa del sistema público de salud, en particular de los SESA, y la separación de funciones como mecanismos importantes para lograr los objetivos del sistema. La separación de funciones es vista por las autoridades gubernamentales federales como un mecanismo que facilita: la definición de prioridades; la distribución equitativa del financiamiento para la atención médica; la generación de información y evidencias para la toma de decisiones; el monitoreo y evaluación de desempeño de los servicios y programas, y la rendición de cuentas. ${ }^{14}$ Estos elementos fueron considerados como indicadores de la separación de funciones en los REPSS y es a partir de ellos que se presentan los resultados. En el cuadro I se resume la estructura que soporta la separación de funciones en el SPSS.

\section{Rectoría}

Durante los últimos años se han presentado ajustes importantes, tanto en el marco normativo, como en aspectos instrumentales para lograr un apropiado nivel de organización y funcionamiento del SPSS.

En el primer caso, Campeche y el Distrito Federal (DF), reformaron sus leyes estatales de salud en $2008 \mathrm{y}$ 2009, respectivamente, adicionando un capitulado específico sobre PSS y en Jalisco modificaron la condición jurídica del REPSS, que pasó de estructura dependiente del SESA a organismo desconcentrado, lo que presupondría mayor autonomía técnica y operativa para la

\footnotetext{
* Ley General de Salud, Reglamento de la Ley General de Salud en materia de Protección Social en Salud; Reglamento Interno de la Comisión Nacional de Protección Social en Salud; Plan Nacional de Salud 2007-2012.
}

\section{Cuadro I}

\section{Separación de funciones en el Sistema de Protección Social en Salud}

\section{Rectoría}

Secretaría Federal de Salud (SSA) en coordinación con las secretarías estatales de salud (SESA).

\author{
Financiamiento \\ Público (federación y estados)
y aportación familiar. \\ Público (federación y
y aportación familiar. \\ Prestación de servicios \\ Red pública de los Servicios Estata- \\ les de Salud (SESA) y prestadores \\ privados.
}

Evaluación y monitoreo
Comisión Nacional de Protección Social en Salud.

Fuente: Elaboración de los autores a partir de la información de fuentes secundarias. Estudio realizado en el Instituto Nacional de Salud Pública, México, noviembre 2009

SESA: Servicios Estatales de Salud 
administración y gestión de los recursos. Los demás estados estudiados habían reformado con anterioridad sus leyes estatales de salud para incorporar la PSS. De los nueve REPSS evaluados, uno tiene estatus jurídico descentralizado (Baja California), tres desconcentrado (Hidalgo, Jalisco y Morelos) y los cinco restantes pertenecen a la estructura de las Secretarías Estatales de Salud (DF, Querétaro, Guerrero, Campeche, Zacatecas). De acuerdo con los hallazgos del estudio, la naturaleza jurídica por sí misma no dota a los REPSS de autonomía técnica y operativa, más bien está condicionada a otro tipo de factores como la relación entre funcionarios de nivel directivo (REPSS/SESA/Secretario de Salud), la capacidad gerencial de estos funcionarios, el nivel de conocimiento de la normatividad y los procesos del SPSS y la pertenencia al mismo grupo político. Por ejemplo, mientras el REPSS de Querétaro pertenece a la estructura de la Secretaría Estatal de Salud (lo cual teóricamente implica menor autonomía administrativa), según los testimonios recabados en el REPSS y en los SESA de esta entidad federativa, esta condición representa notables ventajas para el manejo de los recursos, la participación en la toma de decisiones y el control financiero. En contraste, el REPSS de Baja California, con estructura descentralizada (lo cual implica total autonomía administrativa y financiera) presenta limitaciones para el manejo de los recursos financieros, así como para la compra de servicios de salud, dado que el responsable del REPSS pertenece a una corriente política diferente al director del SESA, lo cual interfiere en la toma de decisiones.

En el ámbito instrumental fue donde se presentaron los cambios más importantes encaminados, primordialmente, a mejorar la suscripción de acuerdos, convenios y contratos, como es el caso de la incorporación del Anexo IV a los Convenios de Coordinación. La finalidad fue establecer criterios y topes de asignación presupuestal para mejorar el uso de los recursos financieros del sistema, dado que había entidades federativas que dedicaban hasta 70\% del presupuesto al pago de personal o a la compra de medicamentos. Además se ajustaron y actualizaron manuales de funciones y procedimientos en diversas áreas, con vistas a mejorar la organización y operación de los REPSS.

Pese a los avances encontrados, existe una idea generalizada en la mayoría de los informantes entrevistados de la persistencia de dificultades en la adecuación del marco jurídico-normativo federal y estatal, lo que permite discrepancias en la interpretación de las normas entre autoridades estatales REPSS/SESA; falta de claridad, concordancia y precisión de la reglamentación e instrumentación de este marco para el cumplimiento de los objetivos (diversos lineamientos tienen el carácter de recomendación, por lo cual no son de obligatorio cumplimiento y los estados pueden o no acogerse a ellos) y escasa reglamentación para la suscripción de contratos con prestadores privados (cuadro II).

\section{Cuadro II}

Dificultades EN LA ADECUACIÓn DEL MARCo JURídico-NORMATIVO FEDERAL Y ESTATAL del Sistema de Protección Social en Salud señaladas por los informantes de las entidades federativas

\begin{tabular}{|c|c|c|}
\hline Obstáculos marco normativo & Descripción & Entidades federativas \\
\hline \multirow{3}{*}{ Vacíos } & Falta de lineamientos para la compra de servicios de salud a privados & DF, Baja California \\
\hline & $\begin{array}{l}\text { La normatividad no precisa la naturaleza jurídica que deben tener los REPSS ni su estructura } \\
\text { orgánica, lo cual origina que cada estado los defina bajo sus propios criterios. }\end{array}$ & Hidalgo, Guerrero \\
\hline & $\begin{array}{l}\text { Ausencia de facultades y mecanismos para sancionar el incumplimiento de los prestadores de } \\
\text { la red pública de servicios por parte de los REPSS }\end{array}$ & Morelos \\
\hline $\begin{array}{l}\text { Discrepancias en la } \\
\text { interpretación y aplicación } \\
\text { de la normatividad }\end{array}$ & $\begin{array}{l}\text { Injerencia del REPSS en la acreditación de las unidades de salud. } \\
\text { Divergencias en la observancia del Anexo IV del Convenio de Coordinación referente a las } \\
\text { partidas y techos presupuestales autorizados para el gasto. }\end{array}$ & $\begin{array}{l}\text { DF, Hidalgo, Baja Califor- } \\
\text { nia, Guerrero }\end{array}$ \\
\hline Variaciones & $\begin{array}{l}\text { Cambios y ajustes en la normatividad y lineamientos operativos. Esto trae como consecuencia } \\
\text { discrepancias en la interpretación entre autoridades federales y estales y entre funcionarios del } \\
\text { REPSS y los SESA. }\end{array}$ & Morelos, Querétaro \\
\hline Rigidez normativa & $\begin{array}{l}\text { Para la aplicación del gasto (Anexo IV del Convenio de coordinación). } \\
\text { Para la compra de equipos, insumos y medicamentos }\end{array}$ & Baja California, Zacatecas \\
\hline
\end{tabular}

Fuente: Información primaria recolectada durante el trabajo de campo en las entidades federativas para efectos de la evaluación del SPSS, 2009

REPSS: Regímenes Estatales de Protección Social en Salud

SESA: Servicios Estatales de Salud 
En materia de evaluación, monitoreo y seguimiento, también se identifican avances. Uno de ellos lo constituye la verificación del cumplimiento de los acuerdos de gestión y contratos que se realiza mediante diversos mecanismos como la comprobación del gasto, la evaluación de indicadores de desempeño, el cumplimiento de metas y el trámite de quejas. Pese a ello persisten dificultades tanto para disponer de información actualizada, como para realizar una rendición de cuentas expedita y oportuna. Estos problemas se encuentran distribuidos a lo largo de la cadena de captación, procesamiento, análisis y difusión de la información (cuadro III).

\section{Financiamiento}

Separación de funciones entre autoridades de salud $y$ agencias financieras

De acuerdo con informantes del ámbito federal y con el Reglamento de la Ley General de Salud en materia de Protección Social en Salud, el SPSS promovió dos cambios esenciales: en la estructura financiera del subsector público y en la gestión y administración de los recursos en el ámbito estatal.

El primer cambio fue dotar de recursos frescos a los estados a partir de la redistribución de recursos públicos a través de la cuota social y las aportaciones solidarias realizadas tanto por la federación como por los estados. El segundo fue en la gestión y administración de los recursos financieros que quedó a cargo de los REPSS. Las normas establecen que la transferencia de recursos a los estados debe realizarse trimestralmente. Sin embargo, una problemática expresada frecuentemente por funcionarios del ámbito estatal (DF, Morelos, Hidalgo, Guerrero, Querétaro, Baja California y Jalisco) es el retraso en la transferencia desde la Comisión hacia la entidad federativa. Los casos más extremos se encontraron en Guerrero y Baja California, con retardos entre 6 y 9 meses. Este problema es visto de manera antagónica por los actores del ámbito federal y estatal. Desde el punto de vista de los primeros, se debe primordialmente al incumplimiento (en tiempo y forma) de uno o algunos de los requisitos que debe acatar la entidad federativa: acreditación de la aportación solidaria estatal, comprobación de la ejecución del gasto o porque han incurrido en gastos no contenidos en el CAUSES, por lo que deben realizar las aclaraciones pertinentes o devolver el dinero. Por su parte, funcionarios del ámbito estatal atribuyen este retraso a la CNPSS, argumentando la tardanza para validar el padrón de afiliados, la burocratización en el proceso de certificación de la comprobación de gastos y la falta de comunicación y coordinación entre la Dirección General de Afiliación y la Dirección General de Financiamiento.

Una vez que llegan los recursos financieros a la entidad federativa, el tiempo de transferencia al interior de las dependencias estatales es muy variable, lo cual puede deberse a causas de tipo político (lucha de poder entre partidos, fracciones y grupos, desvío de recursos por compromisos pactados, proselitismo político); de transparencia en el uso de recursos (cubren otros gastos con los recursos del SPSS y dejan descubierta el área donde se generó el recurso); de trámites burocráticos innecesarios (uso de tiempos excesivos para el traslado de los recursos a las áreas de demanda), y falta de capacitación del personal de la secretaría de finanzas del

\section{Cuadro III \\ Problemas en el sistema de información para la Rendición de cuentas}

Proceso

a) Captación

b) Procesamiento

Problemática

- Multiplicidad de mecanismos e instrumentos para el registro de la información sobre volumen y tipo de servicios prestados

- Retraso tecnológico para el acopio y procesamiento de la información

- Escasez de mecanismos para exigir a los SESA información oportuna, veraz y confiable para realizar la comprobación del gasto

- Retraso en el procesamiento de los datos por diversas causas: falta de oportunidad en la entrega de la información por las unidades de salud, jurisdicciones sanitarias y/o hospitales, retraso tecnológico, escasez de personal, multiplicidad de formatos para consolidar la información

- Duplicación de información para diferentes dependencias, tanto del ámbito estatal como federal

- Retraso de los REPSS en la revisión y validación de información para conciliar dudas y aclaraciones antes de enviarla a la Comisión c) Análisis y difusión • Escasez de personal

- Retrasos en la validación de la información por parte de la Comisión

d) Otros - Falta de claridad sobre el tipo de información y la dependencia a la cual debe enviarse

Fuente: Información primaria recolectada durante el trabajo de campo en las entidades federativas para efectos de la evaluación del SPSS, 2009

SESA: Servicios Estatales de Salud

REPSS: Regímenes Estatales de Protección Social en Salud 
estado (desconocimiento de los procedimientos y / o la dependencia a la que deben ir los recursos).

\section{Prestación de servicios}

Separación de funciones entre autoridades de salud y proveedores de servicios

El SPSS concibe la separación de funciones como una relación contractual: la entidad financiadora -en este caso los REPSS- compra servicios de salud a la red de prestadores públicos a través de la firma de acuerdos de gestión y a la red de proveedores privados con base en contratos de prestación de servicios. La suscripción de estos documentos jurídicos se convierte en el instrumento clave para asignar y transferir recursos en función del número de afiliados y de la cantidad y tipo de servicios prestados acordes con la oferta de servicios del Sistema. ${ }^{15}$

En la práctica, la mayoría de los REPSS compran servicios de salud a proveedores de la red pública (SESA) como primera opción (y en ocasiones la única). Incluye servicios ambulatorios y hospitalarios de primer y segundo nivel de atención. Según lo indicado por los entrevistados del ámbito estatal, los acuerdos de gestión se firman anualmente, aunque con frecuencia se presentan retrasos asociados a burocratización y exceso de trámites, lo cual genera demoras en la transferencia de los recursos. De los nueve estados estudiados sólo dos compraban servicios de salud a prestadores privados (Jalisco e Hidalgo). El volumen de esta subrogación es muy bajo en razón a que priorizan la oferta de la red pública.

Una problemática identificada en la mayoría de los estados estudiados es que los recursos financieros del sistema son destinados a incrementar el techo presupuestal de los SESA (del cual depende la red pública de prestadores de servicios) sin un análisis previo de las necesidades de la población afiliada y de la capacidad de oferta de las unidades de atención, lo cual trae como consecuencias saturación de servicios en algunas áreas y subutilización en otras.

En cuanto a la transparencia y uso de los recursos, en teoría la normatividad vigente le proporciona blindaje al sistema para impedir manejos inadecuados en el uso de los recursos financieros y establece topes de gasto para evitar desvíos e ineficiencias. Sin embargo, informantes del ámbito estatal y federal coinciden en señalar problemas de ineficiencia (baja productividad de las unidades de salud, altos costos de producción de servicios, duplicidad de funciones REPSS /SESA, aumento de techo presupuestal para las unidades de salud sin tomar en cuenta su desempeño), falta de transparencia en el uso de los recursos (retraso en la ejecución presupuestal sin causa aparente, compras sin procesos previos de licitación, financiamiento de servicios no contenidos en el CAUSES, concentración en el manejo de recursos que permite la discrecionalidad en las decisiones por grupos restringidos de personas, contratación de personal por preferencias personales, recomendación o compromiso político y no por perfil técnico) y escasa capacidad administrativa y gerencial de muchos de los directores de hospitales y de unidades de salud y de algunos funcionarios de alto nivel y mandos medios de los REPSS.

\section{Discusión}

Dada la naturaleza de la evaluación (procesos gerenciales) el alcance del estudio no permitió identificar efectos de la separación de funciones sobre la organización y desempeño los servicios de salud en términos de cobertura, accesibilidad, eficiencia, calidad y/o equidad de la atención. Sin embargo, los hallazgos permiten señalar que el SPSS está transitando hacia la separación de funciones, dado que normativa y operativamente el control de la rectoría, la regulación y el financiamiento permanecen a cargo de las entidades creadas para ese fin (CNPSS en el ámbito federal y REPSS en el ámbito estatal), mientras que la prestación de los servicios se abre a la participación plural de prestadores públicos y privados (éstos últimos en menor grado).

En este sentido, existen claros indicios de separación de funciones en cada una de las nueve entidades federativas estudiadas. Esto se identifica a partir de su capacidad para administrar los recursos financieros del sistema, de comprar servicios de salud mediante la suscripción de acuerdos de gestión con la red pública de servicios y de contratos con proveedores privados y de realizar evaluación y monitoreo de las acciones ejecutadas. Sin embargo, el avance es heterogéneo entre las diversas entidades federativas, según las condiciones específicas de cada estado, tales como la tecnocracia presente, la capacidad gerencial y técnica de los funcionarios de alta dirección en los REPSS y los SESA y los recursos financieros y humanos disponibles. Bajo este contexto estados como Jalisco, Campeche y Zacatecas presentan mayores avances hacia la separación de funciones, mientras Guerrero y el DF son los que presentan procesos más lentos.

A pesar de estos avances, la evaluación logró identificar problemas normativos, técnicos, políticos y gerenciales para gestionar y administrar de manera adecuada los recursos financieros del sistema y para coordinar de manera oportuna, eficiente y sistemática la provisión de los servicios de salud (cuadro IV). Son escasos los mecanismos con los que cuentan los REPSS para ofrecer incentivos a los prestadores de la red pú- 


\section{Cuadro IV \\ Problemas para avanzar en la separación de funciones}

\begin{tabular}{|c|c|c|}
\hline Problemas & Descripción & Entidades federativas \\
\hline Normativos & $\begin{array}{l}\text { Vacíos regulatorios, discrepancias en la interpretación y aplicación de la normatividad, varia- } \\
\text { ción en la observancia de la ley, los reglamentos y lineamientos y rigidez normativa }\end{array}$ & $\begin{array}{l}\text { DF, Baja California, Hidalgo, Guerrero, } \\
\text { Morelos, Querétaro, Zacatecas. }\end{array}$ \\
\hline Técnicos & $\begin{array}{l}\text { Desconocimiento del marco normativo y operativo del sistema por parte de funcionarios } \\
\text { de la Secretaría de Finanzas del estado, SESA y REPSS }\end{array}$ & DF, Jalisco. \\
\hline Políticos & $\begin{array}{l}\text { Conflicto de poderes entre partidos, fracciones y grupos políticos que participan en las } \\
\text { diversas dependencias del REPSS y de la Secretaría de Salud, búsqueda del control de los } \\
\text { recursos del sistema, desvío de recursos por compromisos pactados, proselitismo político, } \\
\text { injerencia de grupos de interés (proveedores, usuarios) }\end{array}$ & Guerrero, Baja California, Morelos, DF \\
\hline Gerenciales & $\begin{array}{l}\text { Escaso liderazgo, insuficiente capacidad para resolver problemas o mantener una comuni- } \\
\text { cación proactiva con los equipos de trabajo y con diversos funcionarios de nivel directivo y } \\
\text { mandos medios del REPSS }\end{array}$ & $\begin{array}{l}\text { Problema generalizado en las entidades } \\
\text { federativas estudiadas en funcionarios } \\
\text { de diversos niveles de la estructura }\end{array}$ \\
\hline
\end{tabular}

Fuente: Información primaria recolectada durante el trabajo de campo en las entidades federativas para efectos de la evaluación del SPSS, 2009

REPSS: Regímenes Estatales de Protección Social en Salud

SESA: Servicios Estatales de Salud

blica para hacer uso eficiente de los recursos, así como también escasos son los instrumentos que les permiten sancionar el incumplimiento de los acuerdos de gestión suscritos entre autoridades y prestadores dentro de la misma institución. Un elemento que cobra relevada importancia es el factor político. La búsqueda del control de los recursos asignados a los REPSS ha llevado a la mayoría de los estados a mantener estas unidades al interior de su estructura y no buscar desconcentrarlas o descentralizarlas para garantizar su operación sin interferencias externas. Aún en los casos de desconcentración y descentralización fue posible registrar situaciones en las que los directivos del REPSS tuvieron que ceder ante las decisiones de las autoridades de salud.

\section{Conclusión}

La separación de funciones entre el financiamiento y la prestación de servicios está siendo promovida dentro de la operación del Sistema de Protección en Salud en México. Esta separación es clave para lograr los objetivos estratégicos del sistema. Los avances mostrados son claros pero heterogéneos entre los estados donde se enfrentan importantes retos de tipo político por la búsqueda del control de los recursos del sistema, en la falta de experiencia y capacitación de funcionarios para entender y ejecutar la lógica de la operación de los subsidios a la demanda y en la incapacidad para decidir sobre bases técnicas el tipo de prestador de servicios que permitirá garantizar la eficiencia en el uso de recursos disponibles.
Sin embargo, a partir de los resultados logrados a la fecha, es importante fortalecer la separación de funciones dentro del REPSS a través de tres grandes estrategias: a) garantizar la voluntad política de respetar los preceptos técnicos del programa, b) promover la capacitación gerencial y c) dotar con reglamentación específica e información fidedigna para la toma de decisiones operativas.

Declaración de conflicto de intereses: Los autores declararon no tener conflicto de intereses.

\section{Referencias}

I. Londoño J, Frenk J. Pluralismo estructurado: hacia un modelo innovador para la reforma de los sistemas de salud en América Latina. En: Observatorio de la salud. Necesidades, servicios, políticas. Capítulo 14. Frenk J, ed. Primera edición. México, DF: Fundación Mexicana para la Salud, 1997.

2. Sojo A. La garantía de prestaciones en salud en América Latina. Equidad y reorganización de los cuasimercados a inicios del milenio. Serie Estudios y Perspectivas 44. México, DF: CEPAL, 2006.

3. Banco Mundial. Informe sobre el desarrollo Mundial 1993. Invertir en Salud. Washington, DC: Banco Mundial, 1993.

4. Organización Mundial de la Salud. Informe sobre la salud en el mundo. Mejorar el desempeño de los sistemas de la salud. Ginebra: OMS, 2000. 5. Secretaría de Salud/ Instituto Nacional de Salud Pública. Marco de referencia. En: Sistema de Protección Social en Salud. Evaluación de Procesos Administrativos. Primera edición 2008. Cuernavaca: Instituto Nacional de Salud Pública, 2008:II.

6. Nigenda G, González-Robledo LM, Aracena B, Juárez C, Wirtz V, Idrovo AJ, et al. Evaluación de procesos administrativos del Sistema de Protección 
Social en Salud 2009. Resumen Ejecutivo. Cuernavaca, México: Instituto Nacional de Salud Pública, 2010.

7. Decreto de Reforma del Artículo 376 de la Ley General de Salud. Ley General de Salud. Diario Oficial de la Federación 24 de febrero de 2005. 8. Reglamento Interno de la Comisión Nacional de Protección Social en Salud. Diario Oficial de la Federación 27 febrero 2004.

9. Frenk J, González-Pier E, Gómez-Dantés O, Lezana MA, Knaul FM. Reforma integral para mejorar el desempeño del sistema de salud en México. Salud Publica Mex 2007;49 suppl I:S2I-S34.

10. Secretaría de Salud. Financiamiento justo y protección social universal: La reforma estructural del sistema de salud en México. México DF: Secretaría de Salud, 2004.

II. Nigenda G. El seguro popular de salud en México. Desarrollo y retos para el futuro. Nota Técnica de Salud No. 2/2005. Washington: Banco Interamericano de Desarrollo, 2005.
12. Secretaría de Salud. Sistema de Protección Social en Salud. Elementos conceptuales, financieros y operativos. $2^{2}$ edición. Colección Biblioteca de la Salud. México DF: Fondo de Cultura Económica, 2006.

13. Reglamento de la Ley General de Salud en materia de protección social en salud. Última reforma publicada en el Diario Oficial de la Federación 13 de noviembre de 2008.

14. Secretaria de Salud. Programa Nacional de Salud 2007-20I2. Por un México sano: construyendo alianzas para una mejor salud. Primera edición, 2007. México, DF: Secretaría de Salud, 2007:106 y 120.

15. Comisión Nacional de Protección Social en Salud. Manual de Gestores de Servicios de Salud. México DF: Secretaría de Salud, 2009. 